\title{
Notch1 signaling regulates chondrogenic lineage determination through Sox9 activation
}

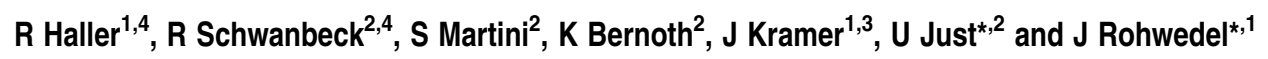

Notch signaling is involved in several cell lineage determination processes during embryonic development. Recently, we have shown that Sox9 is most likely a primary target gene of Notch1 signaling in embryonic stem cells (ESCs). By using our in vitro differentiation protocol for chondrogenesis from ESCs through embryoid bodies (EBs) together with our tamoxifen-inducible system to activate Notch1, we analyzed the function of Notch signaling and its induction of Sox9 during EB differentiation towards the chondrogenic lineage. Temporary activation of Notch1 during early stages of EB, when lineage determination occurs, was accompanied by rapid and transient Sox9 upregulation and resulted in induction of chondrogenic differentiation during later stages of EB cultivation. Using siRNA targeting Sox9, we knocked down and adjusted this early Notch1-induced Sox 9 expression peak to non-induced levels, which led to reversion of Notch1-induced chondrogenic differentiation. In contrast, continuous Notch1 activation during EB cultivation resulted in complete inhibition of chondrogenic differentiation. Furthermore, a reduction and delay of cardiac differentiation observed in EBs after early Notch1 activation was not reversed by siRNAmediated Sox 9 knockdown. Our data indicate that Notch1 signaling has an important role during early stages of chondrogenic lineage determination by regulation of Sox9 expression.

Cell Death and Differentiation (2012) 19, 461-469; doi:10.1038/cdd.2011.114; published online 26 August 2011

The Notch signaling pathway is a highly conserved mechanism of intercellular communication influencing a wide variety of developmental processes throughout embryogenesis and adulthood. ${ }^{1}$ In mammals, one of the five ligands (Delta 1, 3, 4, Jagged1 and Jagged2) binds to one of the four Notch receptors (Notch1-4) on the surface of neighboring cells resulting in a conformational change of the receptor and cleavage of the extracellular domain near the membrane by a metalloprotease of the ADAM (a disintegrin and metalloprotease) family. The ligand-dependent cleavage step renders the truncated receptor sensitive to subsequent intramembrane cleavage by a $\gamma$-secretase multiprotein enzyme complex, thereby releasing the Notch intracellular domain (NICD) into the cytoplasm, allowing it to translocate into the nucleus. There it binds to the transcription factor recombination signal-binding protein for immunoglobulin $\mathrm{J}-\kappa$ (RBP-J, also referred to as RBPJk, CBF1, RBPSUH, SUH or CSL) and assembles into a transcriptional activation complex with co-activator proteins and histone acetylases, finally leading to the expression of downstream target genes. ${ }^{2}$

Several studies have indicated that Notch signaling has an important role during chondrogenesis and cartilage development. $^{3,4}$ Chondrocytes derived from the ectoderm and mesoderm make up cartilage elements during embryogenesis and adulthood. Neural crest cells, which arise from the dorsolateral edge of the neural plate, give rise to the facial element of the skeleton, and sclerotome arising from the paraxial mesoderm gives rise to structures of the axial skeleton. At the initiation of cartilage development, committed progenitor cells aggregate together to form pre-cartilaginous condensations, differentiate into proliferative and prehypertrophic chondrocytes that express cartilage-specific proteins to form the extracellular matrix and finally mature to hypertrophic chondrocytes, which are then replaced by bone. We have shown that cartilage development and enchondral ossification can be recapitulated during EB differentiation of embryonic stem cells (ESCs), ${ }^{5,6}$ thus providing a model for in vitro mechanistic studies.

Components of the Notch signaling pathway are detected at early stages of chondrocyte development ${ }^{7}$ and Notch signaling is active. ${ }^{4}$ Several in vivo studies using models of conditional overexpression of NICD or conditional deletion of Notch core signaling components have further established an important role for Notch signaling in chondrogenesis. ${ }^{8,9}$ Although activation of Notch signaling through a RBP-Jdependent pathway is required for induction of chondrogenesis in both adult and embryonic cells, Notch signaling needs to be inactivated for further maturation and development of the cartilage tissue. ${ }^{10,11}$

\footnotetext{
${ }^{1}$ Department of Virology and Cell Biology, University of Lübeck, Lübeck, Germany; ${ }^{2}$ Department of Biochemistry, Christian-Albrechts-Universität of Kiel, Kiel, Germany and ${ }^{3}$ Medical Department I, University of Lübeck, Lübeck, Germany

${ }^{*}$ Corresponding authors: J Rohwedel, Department of Virology and Cell Biology, University of Lübeck, Ratzeburger Allee 160, D-23538 Lübeck, Germany.

Tel: + 49 451-5004095; Fax: + 49 451-5003637; E-mail: rohwedel@molbio.uni-luebeck.de

or U Just, Department of Biochemistry, Christian-Albrechts-University of Kiel, Olshausenstr. 40, 24098 Kiel, Germany; Tel: + 49 431-880-2513;

Fax: + 49 431-880-2609; E-mail: ujust@ biochem.uni-kiel.de

${ }^{4}$ These authors contributed equally to this work.

Keywords: Notch; chondrogenesis; in vitro differentiation; mouse embryonic stem cells; Sox9; neural crest

Abbreviations: CHX, cycloheximide; EB, embryoid body; ERT, tamoxifen-sensitive human estrogen receptor; ES, embryonic stem; ESC, embryonic stem cell; NICD, Notch intracellular domain; OHT, 4-hydroxy-tamoxifen; NERT, fusion protein of the Notch intracellular domain and the tamoxifen-sensitive human estrogen receptor; PNA, peanut agglutinin; RBP-J, recombination signal-binding protein for immunoglobulin $\mathrm{J}-\kappa$

Received 05.1.11; revised 01.7.11; accepted 22.7.11; Edited by R De Maria; published online 26.8.11
} 
How activated Notch mediates its influences on chondrogenesis at the molecular level is largely unknown. The most well-defined RBP-J-dependent target genes include members of the Hes/Hey family of basis helix-loop-helix transcription factors, which act as transcriptional repressors. ${ }^{12}$ Although Hes 1 was implicated as an important target gene for Notch-mediated mesenchymal progenitor cell maintenance and suppression of chondrogenesis, ${ }^{9}$ it seems not to account for the specification of early progenitor cells towards the chondrogenic lineage. Recently, we have shown that Sox9 is a primary, most likely direct target gene of Notch in ESCs, as well as in ectodermal and mesodermal cells. ${ }^{13}$

Sox9 is a transcription factor which is expressed throughout all cartilage-generating regions and in mesodermal chondrocyte progenitors. ${ }^{14}$ Sox 9 regulates the expression of several cartilage-specific matrix components like collagen II, IX, XI and aggrecan. ${ }^{15-17}$ During transition to the hypertrophic phase, Sox9 expression is downregulated. A heterozygous mutation of the Sox9 gene in humans leads to embryonic dysplasia in particular of the skeleton, known as campomelic syndrome, caused by a reduced Sox 9 dose. ${ }^{18}$ Similarly, heterozygous Sox9 mutants in mice show analogous dysplasia and additionally abnormalities of cartilage progenitors. ${ }^{19}$ In mice, chimera Sox9-deficient cells do not give rise to any cartilage tissue. ${ }^{20}$ Conditional Sox9 knockouts in limb buds before mesenchymal condensations are formed results in the complete absence of cartilage and bones, whereas an inactivation of Sox9 after mesenchymal condensation leads to severe general chondrodysplasia. ${ }^{21}$ Furthermore, it was shown that Sox9 is also involved in the chondrogenic differentiation of neural crest cells, and that all osteo-chondroprogenitors are derived from Sox9-expressing precursors. ${ }^{22,23}$

Despite the uncontested importance of Sox9 for determination and differentiation of chondrocytes and osteocytes, the regulation of Sox9 expression remains rather unclear. Recent evidence suggests that Sox9 is regulated by Hox genes during limb bud development. ${ }^{24}$ Besides cell-intrinsic and cell typedependent transcription factors, cell-extrinsic signals are of special importance for chondrocyte differentiation. Our analyses of in vitro differentiated Sox9 knockout embryonic stem (ES) cells clearly confirm this, as the function of Sox 9 was not cell autonomous during mesenchymal condensation formation. ${ }^{25}$

To examine the role of Sox9 for Notch1-induced effects during chondrocyte development, we used our recently generated murine ES cell line EB5-NERT ${ }^{26}$ that expresses a 4-hydroxy-tamoxifen (OHT)-inducible form of the Notch1 intracellular domain. In the absence of $\mathrm{OHT}$, the fusion protein $\left(\mathrm{NERT}^{27}\right)$ consisting of NICD and the tamoxifensensitive human estrogen receptor (ERT) is kept in the cytoplasm by binding of HSP90 to the ERT part of the protein (Figure 1a). Upon addition of OHT, the NERT fusion protein translocates to the nucleus and transactivates Notch1 target genes by binding to the transcriptional repressor RBP-J and converting it into a transcriptional activator (Figure 1a and Supplementary Video S1). ${ }^{27}$ This system has the advantage that effects of Notch signaling can be studied in a well-defined temporarily regulated manner and in the absence of protein synthesis. EB5-NERT cells were differentiated through EBs, and Notch1 signaling was activated by $\mathrm{OHT}$ at specific time points during EB development. Transient activation of Notch1 signaling during early stages of cell commitment resulted in upregulation of Sox 9 and induction of chondrogenic differentiation. Notch1-induced chondrogenic differentiation was specifically reversed to wild-type levels by knocking down the Sox9level using siRNA targeted against Sox9. Our results show that Sox9 is a critical mediator of Notch1-induced commitment towards the chondrogenic lineage.

\section{Results}

Transient Notch1 signaling at an early stage of ESC differentiation induces chondrogenesis. To investigate the role of Notch1 activation during chondrogenic differentiation of ES cells in vitro, EB5-NERT ES cells that express an OHT-inducible form of the Notch1 intracellular domain (Figure 1a and Supplementary Video S1) were grown for 2 days in hanging drops (0-2 days) for embryoid body (EB) formation. EBs were further cultivated 3 days in suspension (2-5 days), plated at 5 days and cultured for another 34 days $(5+34$ days). Notch1 signaling was temporarily activated by $\mathrm{OHT}$ application during different time windows (0-2, 2-5 days) or continuously from day 2 up to 34 days after EB plating ( $2-5+34$ days). Transient Notch1 activation during early stages of cultivation before EB plating (0-2 and 2-5 days) resulted in an increased number of cartilage nodules from $5+28$ up to $5+34$ days as indicated by Alcian blue staining (Figure 1b). During the first 28 days after EB plating, only a small number of nodules was detectable and the number of nodules strongly increased at $5+34$ days. The induction of Notch1 signaling from 0 to 2 days led to earlier formation and slightly higher numbers of cartilage nodules compared with induction from 2 to 5 days (Figure 1b). In contrast, continuous Notch1 activation completely inhibited the formation of Alcian blue-positive cartilage nodules during the entire differentiation period (Figure 1b). Immunostaining for collagen type II, the characteristic cartilage matrix protein, confirmed that during in vitro differentiation of NERT ES cells, cartilage nodules developed in control (Figure 1C, I-III) and transiently Notch1activated EB cultures, from 0 to 2 days (Figure 1C, IV-VI) and 2-5 days (Figure 1C, VII-IX), respectively, whereas only collagen type II-positive fibers but no nodules were found when Notch1 was activated continuously (Figure 1c, X-XII). As the highest induction of chondrogenic differentiation was achieved when Notch1 was activated from days 0 to 2 during EB differentiation, during early commitment, the following experiments were carried out under these conditions.

Induction of chondrogenesis by activated Notch1 is mediated by upregulation of Sox9. Recently, we have shown that Notch1 signaling upregulates Sox9, an essential transcription factor involved in chondrogenesis, in ESCs and ectodermal, as well as in mesodemal progenitor cells. ${ }^{13}$ Thus, we reasoned that Sox 9 may be involved in the induction of chondrogenesis by activated Notch1. To analyze this further, we first determined induction of Sox9 expression by Notch1 during EB differentiation. In line with our previous results, ${ }^{13}$ we found that Notch1 activation resulted in immediate upregulation of Sox9 expression in 
a NERT

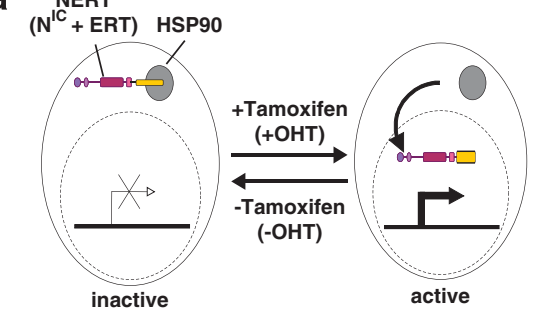

b

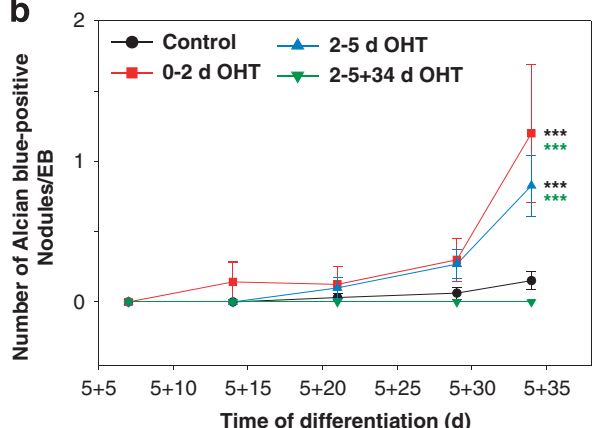

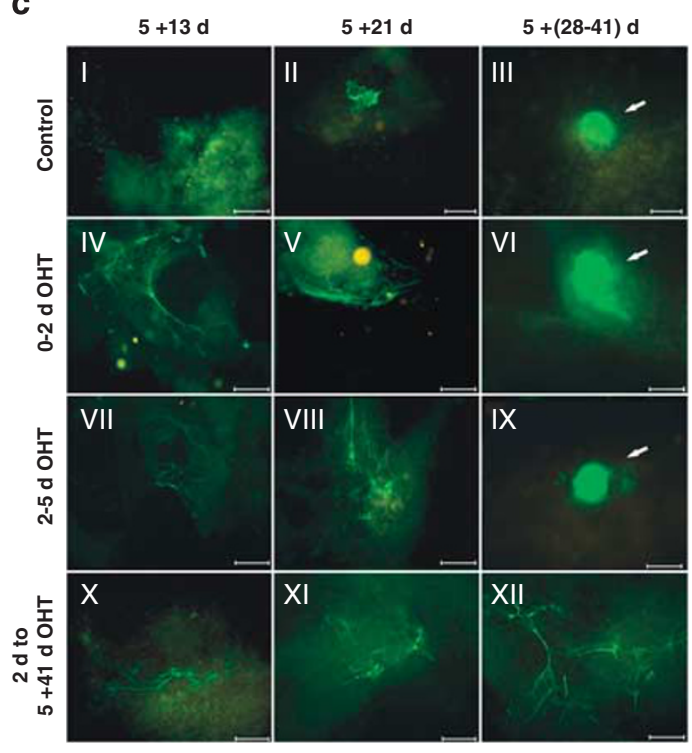

Figure 1 Chondrogenic ES cell differentiation is induced by transient Notch1 signaling. (a) Conditional activation of the constitutive active Notch1 intracellular domain (NICD) using the tamoxifen-inducible system. In the absence of tamoxifen (OHT), Notch signaling is inactive in cells stably expressing a fusion protein consisting of NICD and the hormone-binding part of the tamoxifen-sensitive human estrogen receptor (ERT) because the fusion protein NERT is kept in the cytoplasm by binding of HSP90 to the ERT part of the protein. Upon addition of OHT, the NERT fusion protein translocates to the nucleus and transactivates Notch1 target genes by binding to the transcriptional repressor RBP-J and converting it to a transcriptional activator. An animated version of the inducible Notch1 system can be found in the Supplementary Material Video 1. (b) NERT ESCs were differentiated in vitro as EBs and Notch1 signaling was activated by the addition of $\mathrm{OHT}$ during $0-2$ days, 2-5 days or continuously from day 2 during EB culture. EBs were analyzed for the formation of cartilage nodules by Alcian blue staining. Transient Notch1 activation induced chondrogenic differentiation at $25-34$ days after plating of the 5 -day-old EBs $(5+25$ to $5+34$ days). In contrast, continuous Notch1 activation $(2-5+34$ days) completely inhibited chondrogenic differentiation. The error bars indicate S.E.M. from three independent experiments. Two-way ANOVA revealed highly significant interactions and variabilities with $P$-values $<0.0001$. Significant differences determined by Bonferroni's post-test in comparison with the uninduced (control) NERT EBs (black asterisk) and with the continuously OHT-induced NERT EBs (green asterisk) are ${ }^{\star \star} P<0.01,{ }^{* \star \star} P<0.001$. (c) Immunostaining for collagen type II confirmed the results. Collagen type II-positive nodules were detected during in vitro differentiation of NERT ES cells after early Notch1 activation (0-2 days OHT, 2-5 days OHT) and in the control from 28 up to 41 days after EB plating (5+ (28-41) days). In contrast, only collagen type II-positive fibers but no nodules appeared after continuous Notch1 activation in EBs from 2 days before plating up to 41 days after EB plating (2 days to $5+41$ days). $\operatorname{Bar}=100 \mu \mathrm{m}$

ESCs also in the presence of the protein synthesis inhibitor cycloheximide $(\mathrm{CHX})$ used at a concentration and under conditions previously established to inhibit protein synthesis (Figure 2a), suggesting Sox9 to be directly targeted by Notch signaling. Supporting this idea, we have recently shown the potential binding sites of RBP-J within the Sox9 promoter. ${ }^{13,28}$ Sox 9 was highly inducible by activated Notch1 during early EB differentiation, but not at late time points of differentiation (day 10; Figure 2b). Furthermore, to show the direct nature of Sox9 regulation by Notch1, we used luciferase reporter constructs that were transiently transfected into ES cells. To this end, we mutated the two potential RBP-J-binding sites located approximately $+40 \mathrm{bp}$ (BS1) and -325 bp (BS2) relative to the transcription start site of Sox9 (Figure 2c). When BS1 was mutated, the basic expression of the reporter (i.e., $-\mathrm{OHT}$ ) was increased, most likely driven by the lack of repression of RBP-J. At the same time, the expression upon Notch1 activation was reduced resulting in a decreased induction compared with the wildtype Sox9 promoter (Figure 2c). The same overall effects, but even stronger, were observed when BS2 was mutated (Figure 2c). However, a slight inducibility of the Sox9 promoter remained in both mutants, even if this did not seem to be statistically significant. Taken together, these results revealed an important role for both RBP-J-binding sites in Notch1-mediated Sox9 upregulation, possibly acting in a synergistic manner.

Next, we sought to dissect the role of Notch1-induced upregulation of Sox9 expression during chondrogenic differentiation from ESCs. To this end, we used a siRNAmediated Sox9 knockdown to control Sox9 levels, which resulted in decreased Sox9 expression close to control levels (Figure 3). Manipulating the Sox9 expression levels back to control levels during Notch1 activation at an early stage of EB cultivation (0-2 days) had a strong influence on the formation of cartilage nodules in EBs from $5+14$ days up to $5+49$ days as analyzed by Alcian blue staining and realtime PCR for collagen type // (Figure 4). After Notch1 activation, the number of nodules increased significantly in comparison with the control after EB plating (Figure 4a). Knockdown of Sox 9 expression reversed this inductive effect back to control levels during the same time periods (Figure 4a), reaching values close to normal levels of chondrogenic differentiation. Real-time PCR for collagen type II mRNA during EB differentiation showed that activation of Notch1 signaling during days $0-2$ of EB cultivation resulted in a slight upregulation of collagen type // at the cultivation time when most nodules were detected ( $5+28$ up to $5+34$ days), which was reverted back to control levels by Sox9-specific siRNA (Figure 4b). 
Induction of mesenchymal condensations by activated Notch1 is mediated by upregulation of Sox9. To test whether induction of chondrogenic differentiation is due to an enhanced number of pre-chondrogenic condensations, we analyzed the formation of condensations by PNA (peanut agglutinin) staining. Pre-chondrogenic condensations were present in all EB cultures mainly at $5+14$ days (Figure $5 a$ ). After $5+21$ days, condensations were no longer unambiguously detectable. When we counted the number of condensations in the control and after Notch1 activation,

a

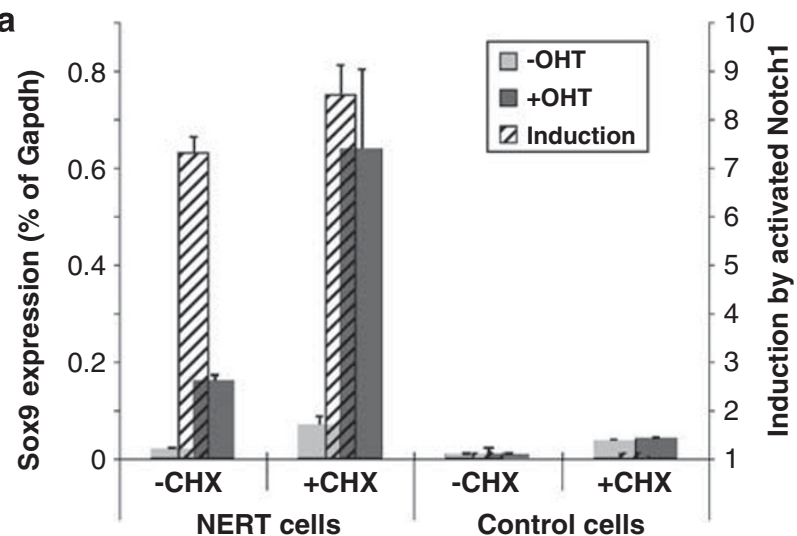

b

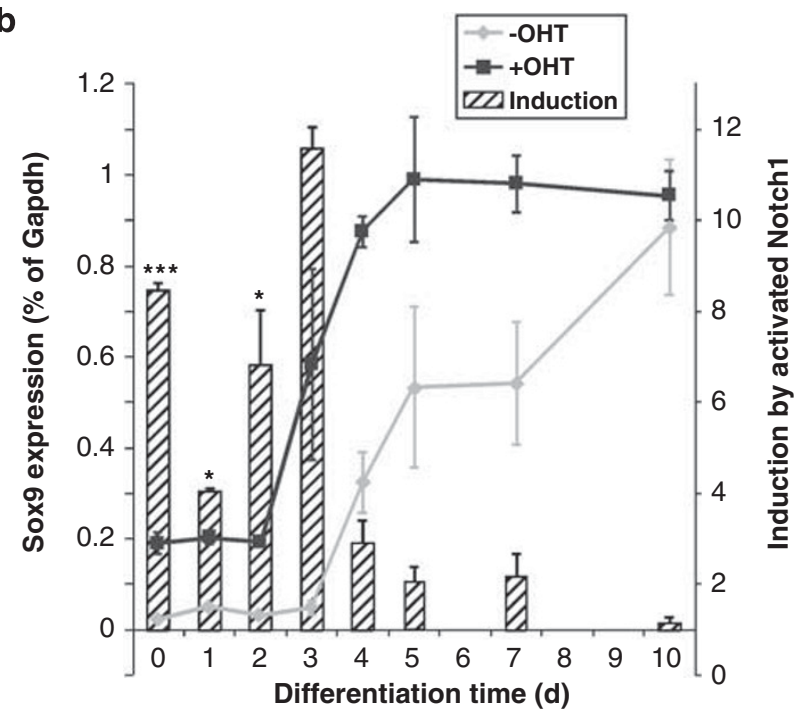

C

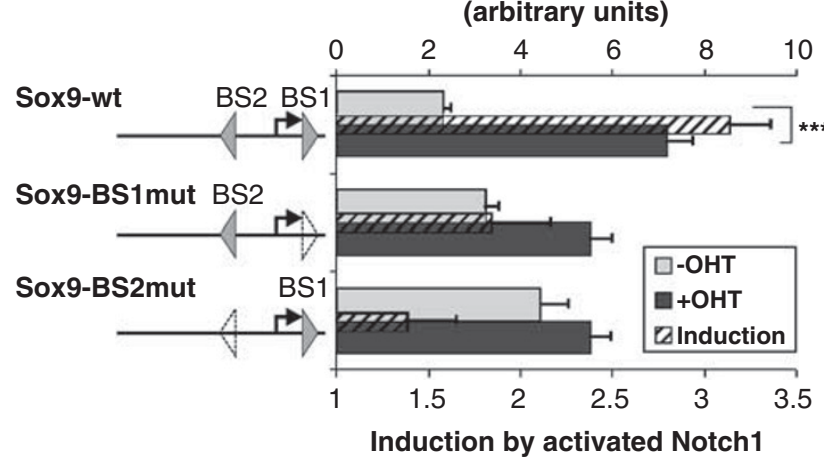

as well as after Sox9 siRNA-mediated knockdown, we found that the number of condensations was clearly enhanced after Notch1 activation and went down to normal numbers after Sox9 knockdown (Figure 5b).

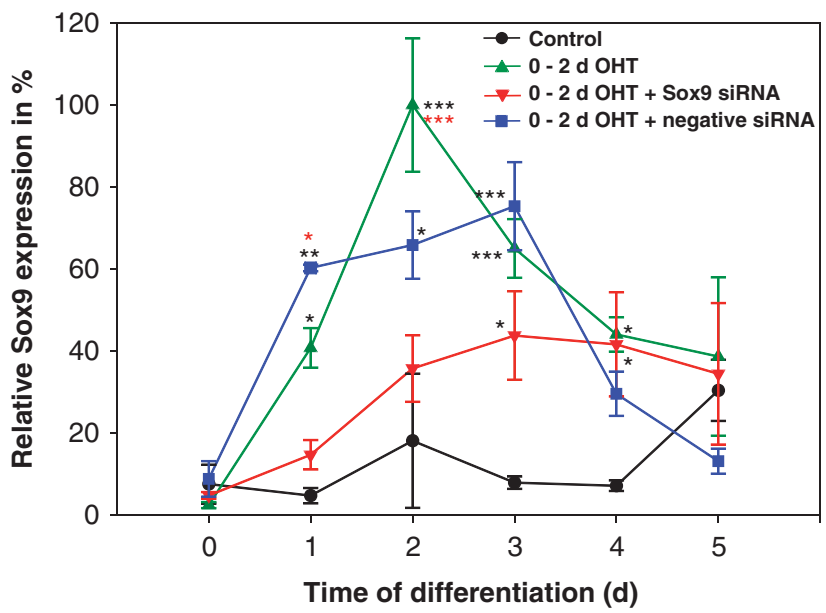

Figure 3 Notch1-induced Sox9 expression is knocked down by application of Sox9 siRNA. Sox 9 expression was measured in NERT EBs cultured for 2 days as hanging drops ( $0-2$ days) and in suspension for additional 3 days ( $2-5$ days). An increase of Sox 9 expression from days 1 to 3 was observed when Notch 1 had been activated from days 0 to 2 . This increase of Sox9 expression was downregulated to almost control levels when cells at day 0 had been transfected with siRNA targeting Sox9. Non-targeting, negative siRNA was used as a control. Real-time PCR results are normalized with Gapdh as internal control and displayed as percentage of maximal Sox 9 expression. The error bars indicate S.E.M. from 3 to 6 independent experiments. Two-way ANOVA revealed highly significant interactions and variabilities with $P$-values $<0.0001$. Significant differences in comparison with the uninduced (control) NERT EBs (black asterisk) and the OHT/Sox9 siRNAtreated NERT EBs (red asterisk) are ${ }^{*} P<0.05,{ }^{* \star} P<0.01,{ }^{* \star \star} P<0.001$ (determined by Bonferroni's post-test)

Figure 2 Sox 9 expression is directly induced by Notch1 activation. (a) Expression of Sox9 is induced in ESCs by Notch1 signaling in the presence of $\mathrm{CHX}$. mRNA levels were measured by qPCR in NERT ESCs. Notch1 signaling was activated in undifferentiated ESCs by adding OHT for $4 \mathrm{~h}$ in the presence or absence of $\mathrm{CHX}$. Deducted inductions of expression are shown by hatched bars. The error bars indicate S.E.M. from 9 and 7 independent experiments for $-\mathrm{CHX}$ and $+\mathrm{CHX}$, respectively. According to ANOVA, the difference between activated and not-activated samples is highly significant not only in general $\left(P<10^{-11}\right)$ but also under $-\mathrm{CHX}\left(P<10^{-9}\right)$ or $+\mathrm{CHX}\left(P<10^{-6}\right)$ conditions alone. Control cells do not show any induction by $\mathrm{OHT}$ in the presence or absence of $\mathrm{CHX}$. (b) Sox9 is induced by activated Notch 1 signaling at early stages of ESC differentiation. mRNA levels were measured by qPCR in differentiating NERT EBs. Notch signaling was activated at days indicated by the addition of $\mathrm{OHT}$ for the last $4 \mathrm{~h}$ of the culture period. ANOVA indicates highly significant differences between $\mathrm{OHT}$-induced and OHT-uninduced (control) samples in general $\left(P<10^{-9}\right)$. Significant statistical differences within 1 day are indicated by ${ }^{\star * \star} P<0.001$, ${ }^{\star \star} P<0.01$ or ${ }^{\star} P<0.05$. (c) Sox9 is induced through RBP-J-binding sites within its promoter. ESCs were transfected with luciferase reporter constructs encompassing the Sox9 wild-type mouse promoter or mutated variants of the RBP-J-binding sites 1 and 2 (BS1mut and BS2mut, respectively). Normalized luciferase activity was measured in the presence or absence of $\mathrm{OHT}$. The deduced induction of activity is shown by hatched bars. Error bars indicate S.E.M. from 10 (wt) or 5 (mutations) independent experiments. Induction of wt Sox9 promoter was highly significant $(P<0.001)$ 

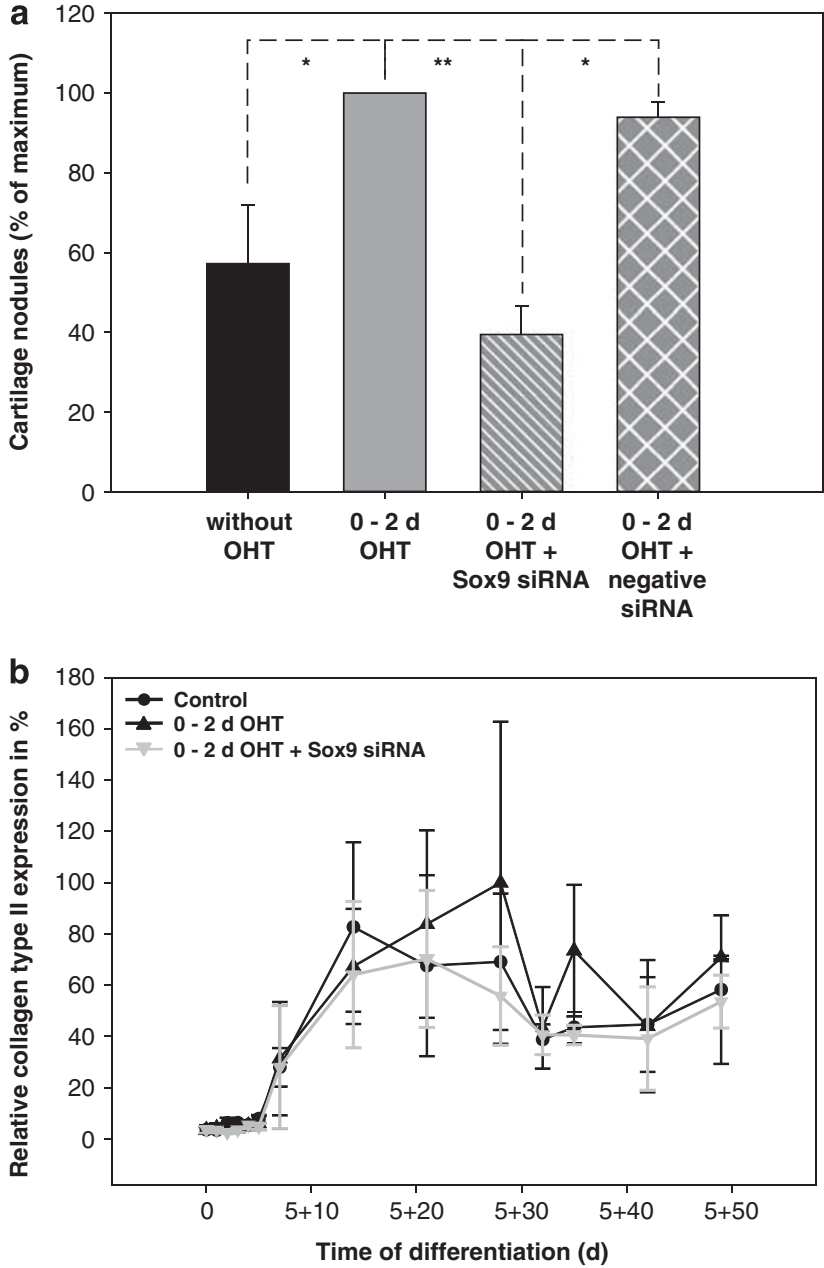

Figure 4 Notch1-induced chondrogenic differentiation is suppressed by siRNAmediated Sox9 knockdown. (a) Notch1 signaling activated by application of OHT from day 0 to day 2 of EB formation (gray bar) enhanced the number of cartilage nodules from 14 days up to 49 days after plating of 5-day-old EBs. This boost of chondrogenic differentiation could be reduced to control levels when cells were transfected at day 0 with siRNA targeted against Sox 9 (hatched bar). Non-targeting, negative siRNA (double hatched bar) was used as a control. The error bars indicate S.E.M. from four independent experiments. One-way ANOVA revealed significant differences between the groups with a $P$-value of 0.0029 . Significant differences determined by Tukey's post-test between the uninduced (control) and OHT-induced NERT EBs, between the OHT-induced and OHT/Sox9 siRNA-treated NERT EBs and between the $\mathrm{OHT} / \mathrm{Sox} 9$ siRNA-treated and the OHT/negative siRNA-treated NERT EBs are: ${ }^{*} P<0.05,{ }^{* *} P<0.01$. (b) A slight upregulation of mRNA expression of the cartilage marker molecule collagen type II at 20,30 and 35 days after EB plating $(5+20,5+30,5+35$ days, respectively) was detected when Notch 1 had been activated by application of OHT from day 0 to day 2 of EB formation. Transfection at day 0 with siRNA targeted against Sox9 resulted in a reduction to control levels, confirming the reversion of chondrogenic differentiation. Real-time PCR results are normalized with Gapdh as internal control and displayed as percentage of maximal collagen type II expression. The error bars indicate S.E.M. from three independent experiments

Notch1-induced Sox9 upregulation is not involved in Notch1-mediated cardiac muscle repression. We along with others have shown earlier ${ }^{26,29}$ that activated Notch1 blocks cardiac muscle differentiation of ESCs. Sox9 is expressed in the developing heart, where it influences a
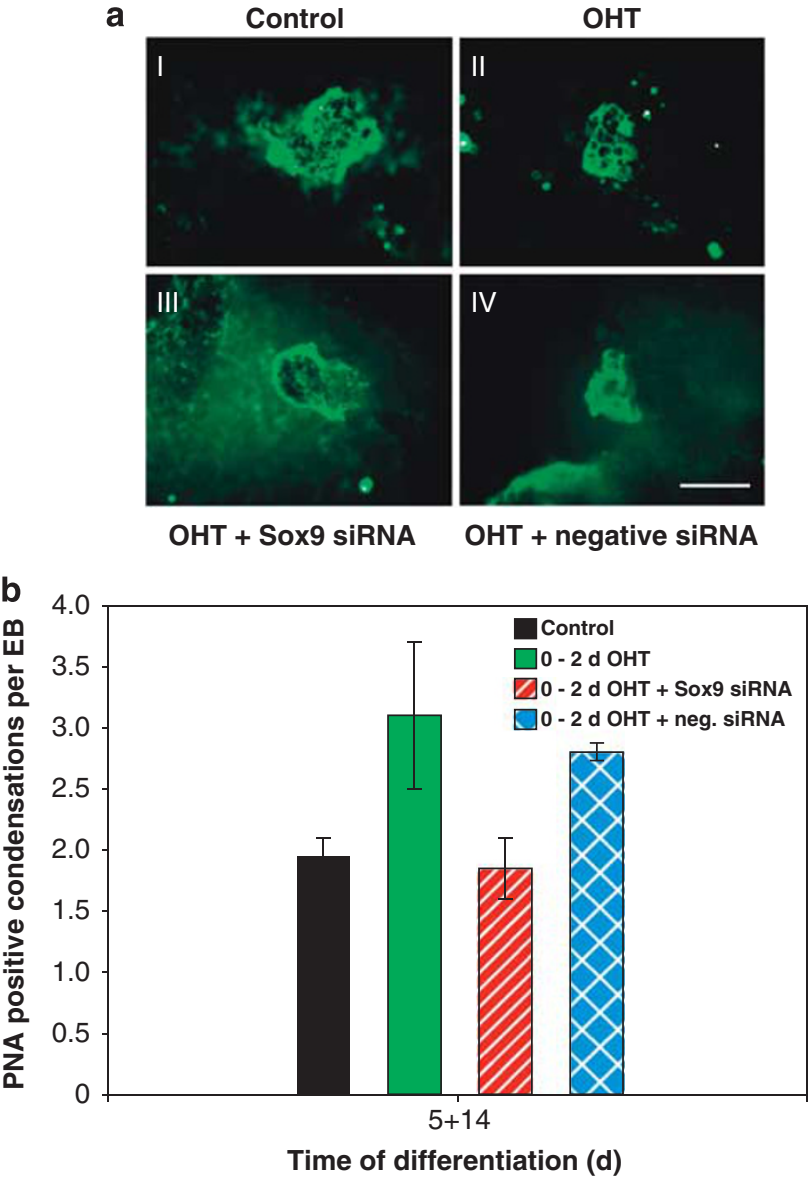

Figure 5 Notch1-induced pre-chondrogenic differentiation is reduced by siRNAmediated Sox9 knockdown. (a) Pre-chondrogenic condensations in NERT EBs 14 days after plating $(5+14$ days) in the absence (I) or presence (II) of OHT and after application of Sox9-specific siRNA (III) and negative (control) siRNA (IV) were detected by PNA staining. (b) The number of condensations developing in NERT EBs 14 days after plating ( $5+14$ days) was enhanced when Notch 1 signaling had been activated by application of $\mathrm{OHT}$ from day 0 to day 2 of $\mathrm{EB}$ formation $(0-2$ days OHT). After siRNA-mediated Sox 9 knockdown at day 0 ( $0-2$ days $\mathrm{OHT}+$ Sox9 siRNA), the number of condensations was reduced to control levels. This effect was not observed when cells were transfected with control siRNA ( $0-2$ days $\mathrm{OHT}+$ negative siRNA). Similar results were obtained at 7 days $(5+7$ days $)$ after plating (data not shown). The error bars indicate S.E.M. from three independent experiments. Bar $=100 \mu \mathrm{m}$

epithelial-mesenchymal transitions and ECM generation but a role for cardiac muscle development has not been described. To analyze whether preventing Notch1-mediated upregulation of Sox9 during early ESC differentiation would specifically affect cardiac differentiation, we knocked down Notch1-induced Sox9 expression and analyzed the differentiating cultures for development of beating cardiac muscle cells. As shown in Figure 6a, activation of Notch1 signaling strongly repressed cardiac muscle differentiation in line with our previous results. In the presence of $\mathrm{OHT}$ to activate Notch signaling throughout the entire differentiation, no beating cardiomyocytes were observed. When Notch1 signaling was temporarily activated from days 0 to 2, cardiac development was severely reduced and delayed for 15 days. Activating Notch1 signaling during days 2 to 5 also 

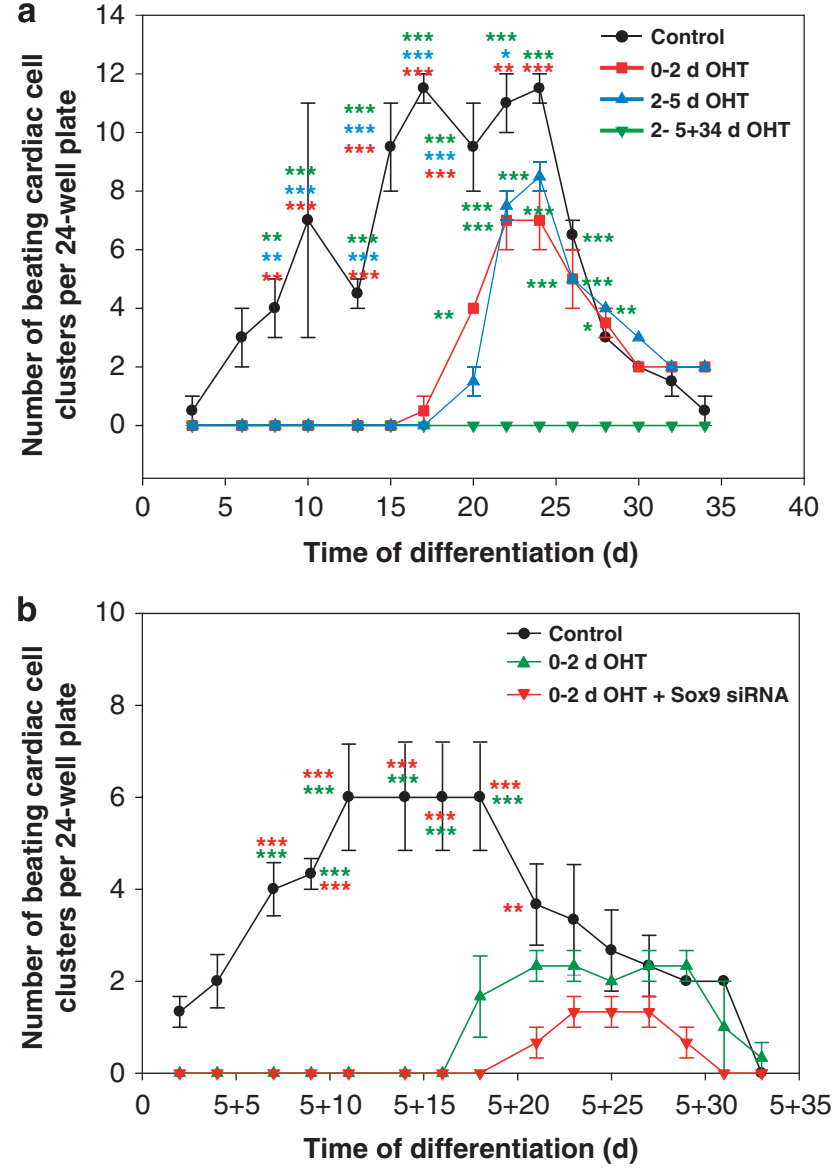

Figure 6 Cardiac muscle differentiation of ESCs in vitro is inhibited by Notch1 signaling and is independent from Notch1-mediated Sox9 induction. (a) Development of cardiac-beating cell clusters in NERT EBs plated at day 5 was completely inhibited when Notch1 had been continuously activated during EB cultivation from 2-day EBs up to 34 days after EB plating (2-5+34 days). When Notch 1 had been activated during the early period of EB suspension culture from 0 to 2 days or 2-5 days, cardiac muscle differentiation was delayed and reduced. The error bars indicate S.E.M. from three independent experiments. Two-way ANOVA revealed highly significant interactions and variabilities with $P$-values $<0.0001$. Significant differences determined by Bonferroni's post-test in comparison with $0-2$ days OHT-treated NERT EBs (red asterisk), 2-5 days OHT-treated NERT EBs (blue asterisk) and continuously OHT-treated NERT EBs (green asterisk) are ${ }^{*} P<0.05,{ }^{* \star} P<0.01,{ }^{* * *} P<0.001$. (b) The delay of cardiac EB differentiation by transient Notch1 activation is not reverted by siRNA-mediated Sox9 knockdown. The delay and reduction of cardiac muscle differentiation of NERT ESCs after Notch 1 activation during the early period of EB suspension culture from 0 to 2 days ( $0-2$ days $\mathrm{OHT}$ ) could not be rescued by targeting Sox 9 with siRNA ( $0-2$ days $\mathrm{OHT}+$ Sox9 siRNA). The error bars indicate S.E.M. from three independent experiments. Two-way ANOVA revealed highly significant interactions and variabilities with $P$-values $<0.0001$. Significant differences determined by Bonferroni's post-test in comparison with OHT-treated NERT EBs (green asterisk), and OHT/Sox9 siRNA-treated NERT EBs (red asterisk) are ${ }^{* *} P<0.01$, ${ }^{* * *} P<0.001$

decreased and further delayed cardiac development (Figure 6a). However, knockdown of Sox9 during Notch1 activation by $\mathrm{OHT}$ did not revert Notch1-induced cardiac muscle repression, suggesting that a Sox9-independent mechanism is involved in mediating the Notch1-induced block of cardiac muscle differentiation (Figure 6b).

\section{Discussion}

In this study, we have provided evidence that Notch1 signaling regulates cartilage formation from ESCs through Sox9. Using an inducible form of activated Notch1, Notch1 signaling was activated at certain stages during in vitro chondrogenic development of ESCs. Continuous Notch1 signaling resulted in a complete block of chondrogenic differentiation, whereas activation of Notch1 signaling at the earliest time of ESC differentiation, when differentiation into the germ layers is initiated, and later during the establishment of the germ layers, caused increased chondrocyte formation. These data suggest that Notch1 signaling influences cell lineage commitment during germ layer and chondrocyte specification, but has a negative influence on chondrogenic differentiation. Early Notch1 activation was paralleled by an increase in Sox9 expression. Knockdown of this early Sox9 expression peak reverted Notch1-induced chondrocyte development back to control levels, arguing that Notch1 regulation of germ layer and chondrocyte specification is mediated by Sox9.

Notch1 activation during ESC germ layer specification enhances ES cell differentiation into the cartilage in vitro. In embryogenesis, chondrocytes arise from sclerotomal cells of the somitic mesoderm, the somitomeres of the hindbrain or mesectodermal cells of neural crest. EBs recapitulate several aspects of early development including germ layer formation and further differentiation into cartilage through the mesoderm and neural crest. $^{6,30}$ Components of the Notch pathway are present in mammalian cells during early stages of embryogenesis ${ }^{31}$ and chondrogenesis, ${ }^{4,7}$ and correct Notch signals are required for normal embryonic cartilage development. ${ }^{8}$ Several in vitro and in vivo studies using gain of function and loss of function reported that increased Notch signaling inhibits chondrogenic differentiation, whereas Notch pathway loss of function results in hypertrophic cartilage and decreased bone formation. 4,8,9,32 In accordance with these reports, continuously activated Notch1 signaling throughout EB differentiation entirely blocked cartilage development. Surprisingly however, transient Notch1 signaling at an early stage of ESC differentiation resulted in an increased chondrocyte development of murine ESCs, whereas in line with our previous work on mesodermal differentiation of $\mathrm{ESCs}^{26}$ cardiac muscle development was severely delayed and decreased. How can this apparent discrepancy be explained? During EB development, recapitulating early embryo formation in vivo, ESCs differentiate within the first day into the primitive ectoderm and then further between days 2 and 5 into the different germ layers. Although cardiac muscle cells are believed to be derived solely from mesodermal cells, chondrocytes are derived from the somitic mesoderm and mesectodermal cells of the neural crest. We along with others have conclusively shown that Notch signaling inhibits mesodermal differentiation and further cardiac muscle differentiation from mesodermal progenitors. $^{33}$ In particular, activation of Notch signaling through inducible expression of the NICD in ES cells inhibits the generation of flk1-positive mesodermal progenitor and 


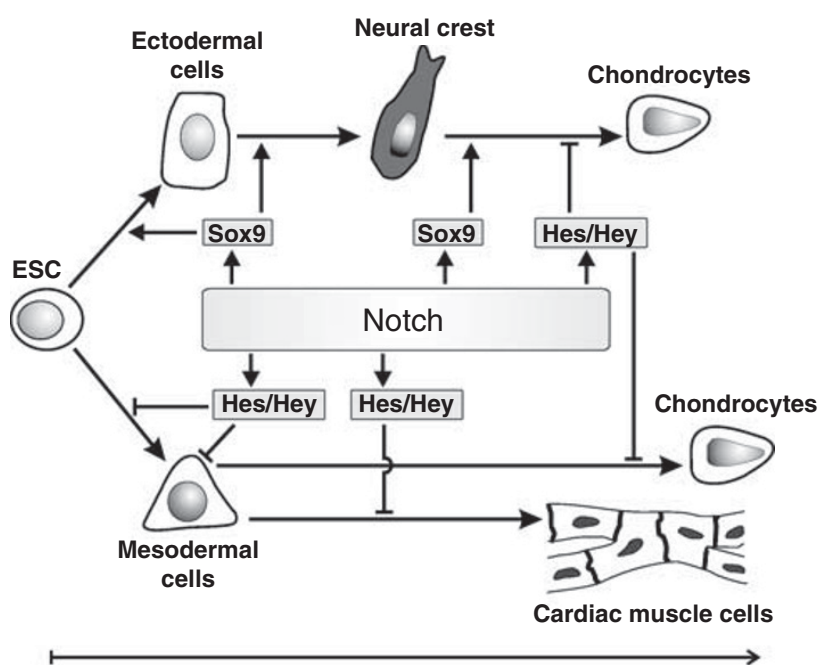

Differentiation

Figure 7 Model for stage-specific regulation of cartilage development by Notch1 signaling during ESC differentiation. Activation of Notch signaling in ESCs induces the expression of Sox9, which directs differentiation first along the ectodermal lineage and then into neural crest precursors and further in chondrogenic precursors. At the same time, Notch signaling represses differentiation of ESCs into the mesodermal lineage, possibly by the Notch target genes of the Hes/Hey family of transcriptional repressors. Terminal differentiation into chondrocytes and cardiac muscle cells is blocked most likely also by induction of Hes/Hey genes

further cardiogenic muscle differentiation. ${ }^{26}$ Vice versa, blockage of Notch signaling in RBP-J-deficient ES cells results in enhanced mesodermal and cardiogenic specification $^{34}$ and expression of a dominant negative mutant of RBP-J, incapable of DNA binding, forces cardiogenesis. ${ }^{29}$ Furthermore, in the presence of appropriate other signals that support neuronal differentiation, Notch signaling drives ESCs towards the neuro-ectodermal lineages. ${ }^{35}$ Within the ectoderm, Notch signaling has a critical role in neural crest formation. ${ }^{36}$ At the neural crest stage, Notch signaling through RBP-J is required for chondrogenic specification and early chondrogenic differentiation. ${ }^{10}$ This idea is supported by real-time PCR results showing an increased expression of the early neural crest marker genes $I d 3$ and Snai1 upon Notch1 activation (Supplementary Figure 1). As Hes/Hey expression can still be activated by Notch1 during later chondrocytic differentiation (Supplementary Figure 2), the suppression of terminal chondrocyte maturation is possibly mediated by Hes/Hey proteins (Figure 7) Taken together, it is thus conceivable that activated Notch1 promotes ectodermal lineage entry in ESCs and further differentiation into neural crest-derived early chondrocytes, while blocking mesodermal and cardiac muscle development (Figure 7).

Notch1-induced chondrogenic lineage determination is mediated through Sox9. Among the genes that are most likely directly activated by Notch 1 signaling in ESCs, we have recently identified Sox9. ${ }^{13}$ This idea of direct regulation of Sox 9 by Notch1 was strongly supported by the luciferase experiments in this study using Sox9 promoter reporter constructs with mutated RBP-J-binding sites. Sox9 is an essential regulator of neural crest development and chondrogenesis and marks the progenitor population of chondrogenic cells. ${ }^{21,37}$ A heterozygous mutation of Sox 9 leads to the human skeletal dysmorphology syndrome, campomelic dysplasia, a very severe form of chondrodysplasia involving defects of all cartilage tissues. ${ }^{18}$ Similarly, skeletal abnormalities including craniofacial abnormalities and growth retardation are found in Alagille syndrome, an autosomal disorder caused by mutations of the Notch ligand Jagged $1 .^{38}$ In line with our previous results, ${ }^{13}$ we have shown here that Notch1 signaling upregulates Sox9 expression in ESCs under differentiation conditions favoring chondrocyte development. Knockdown of this early Sox 9 expression peak reverted Notch1-induced effects on chondrocyte development back to control levels, supporting our model that Notch1 regulation of germ layer and chondrocyte specification is mediated by Sox9 (Figure 7). This view is further supported by recent experiments using an activated form of Notch, the $\gamma$-secretase inhibitor DAPT to block Notch signaling and shRNA against Sox9 to show that Sox9 is regulated by Notch signaling and required for Müller glial cell development in the mouse retina. ${ }^{39}$ Classically, many of the Notch functions are mediated by the Hes (Hairy/ Enhancer of split) and Hey (also called Herp/Hesr/Hrt/CHF/ gridlock) families of basic helix-loop-helix type transcriptional repressors. ${ }^{12}$ Hes and Hey are primary Notch effectors that affect cell-fate decisions by suppression of downstream target genes, such as tissue-specific regulators. ${ }^{12}$ During mesodermal development, Notch signaling as well as Hes and Hey proteins block the generation of mesodermally derived cardiac muscle cells by repressing cardiogenic transcription factors such as GATA4 and GATA6. ${ }^{40}$ Although Hes1 and Hes5 are both dispensable for cartilage formation, ${ }^{41}$ several observations indicate that Hes/Hey proteins negatively influence chondrocyte maturation. Along this line, Hey1 overexpression leads to chondrocyte hypertrophy in bone, ${ }^{42}$ and Hes 1 and Hey 1 suppress COL2A1 expression and chondrogenic differentiation. ${ }^{43}$ Taken together, this suggests that Notch signaling induces Sox9 expression, which promotes and initiates neural crest development and further differentiation into chondrocytes and at the same time induces expression of Hes/Hey transcriptional repressors that inhibit mesodermal and cardiac differentiation and terminal chondrocyte maturation (Figure 7).

Specification of neural crest and further of chondrocytes involves a genetic network including Notch, Wnt/FGF/RA and BMP signaling and transcriptional effectors such as Sox9, Id3 and Snail1 as major determinants. ${ }^{44-46}$ It is interesting that expressions of Id3 and of Snail1 are also regulated by Notch1 signaling, ${ }^{13}$ underscoring the importance of Notch 1 in this process. Although Sox 9 promotes neural crest and chondrocyte specification, Id 3 and Snail1 act as inhibitors, further supporting our recently established model that Notch signaling determines lineage decisions and expansion of stem cells by directly activating both key lineage-specific transcription factors and their repressors. ${ }^{13}$ In conclusion, we propose that Notch signaling induces Sox9 expression, which promotes 
and initiates neural crest development and further differentiation along the chondrocyte lineage (Figure 7).

\begin{abstract}
Materials and Methods
Cell culture and differentiation of EBs. The ES cell line EB5-NERT, ${ }^{26}$ expressing a OHT-inducible form of the Notch1 intracellular domain, was grown in maintenance medium consisting of DMEM (Invitrogen, Karlsruhe, Germany) supplemented with 15\% FCS (Invitrogen), non-essential amino acids (Invitrogen, stock solution diluted 1:100), $2 \mathrm{mM}$ L-glutamine (Invitrogen), $5 \times 10^{-5} \mathrm{M}$ 2-mercaptoethanol (Serva, Heidelberg, Germany) and $5 \mathrm{ng} / \mathrm{ml}$ leukemia inhibitory factor (Millipore, Temecula, CA, USA) as described previously for line D3. ${ }^{6}$ The EB5-NERT clone had been generated from EB5 ES cells. ${ }^{26}$ We have previously shown that in vitro differentiation of control EB5 cells was not affected by OHT application. ${ }^{13}$ For differentiation of EB5-NERT cells, aliquots of $20 \mu$ ldifferentiation medium, consisting of DMEM (Invitrogen) supplemented with 20\% FCS (Invitrogen), non-essential amino acids (Invitrogen, stock solution diluted 1:100), $2 \mathrm{mM}$ L-glutamine (Invitrogen), $5 \times 10^{-5} \mathrm{M}$ 2-mercaptoethanol (Serva) and containing 800 cells were cultivated as 'hanging drops' for 2 days and, after transfer on bacteriological petri dishes, in suspension for additional 3 days. ${ }^{6}$ The 5 -day-old EBs were plated separately onto gelatin (0.1\%)-coated 24-well microwell plates for morphological analysis and Alcian blue staining; for RNA extraction and RT-PCR, 10 EBs were plated onto a $6-\mathrm{cm}$ tissue culture plate; and for immunostaining, 10 EBs were plated onto 2-well $\left(21.3 \times 20 \mathrm{~mm}^{2}\right)$ Lab-Tek chamber slides (Nunc, Wiesbaden, Germany). Alcian blue staining and PNA staining were performed as described previously. ${ }^{6,25}$ Two days after EB plating, the medium was changed to low FCS medium supplemented with $0.2 \%$ FCS instead of $20 \%$. All differentiation experiments were repeated at least three times. To confirm Sox 9 as a direct target gene of Notch1 in mesodermal differentiation of EBs, differentiation was carried out as described previously. ${ }^{13}$ Blocking of protein synthesis was achieved by adding $50 \mu \mathrm{g} / \mathrm{ml} \mathrm{CHX}$ for $4 \mathrm{~h}$.
\end{abstract}

Transfection of EB5-NERT cells. Cells were transfected using $2 \mu \mathrm{l} / \mathrm{ml}$ DharmaFECT 1 transfection reagent (Dharmacon, Lafayette, CO, USA) and a $100 \mathrm{nM}$ pool of siRNA targeting Sox9 (Thermo Scientific, Lafayette, CO, USA) or a $100 \mathrm{nM}$ pool of non-targeting siRNA (Thermo Scientific), so-called negative or control siRNA. The siRNA was incubated with PCR grade $\mathrm{H}_{2} \mathrm{O}$ (Macherey and Nagel, Bonn, Germany) and DMEM (Invitrogen) in the ratio of 1:4:15 for 5 min at room temperature. DharmaFECT 1 transfection reagent (Dharmacon) was incubated with DMEM in the ratio of 1:49 for $5 \mathrm{~min}$ at room temperature. The two solutions were mixed afterwards and incubated for $20 \mathrm{~min}$ at room temperature. A defined number of cells was then cultivated as 'hanging drops' ${ }^{6}$ after application of $\mathrm{OHT}$ (final concentration $1 \mu \mathrm{M}$ ).

Indirect immunostaining. EBs cultivated on chamber slides and rinsed two times with PBS were fixed for $5 \mathrm{~min}$ with methanol:acetone $(7: 3)$ at room temperature, washed three times with PBS again and incubated at $37^{\circ} \mathrm{C}$ for $30 \mathrm{~min}$ with $10 \%$ goat serum. Specimens were then incubated for $1 \mathrm{~h}$ in a humidified chamber at $37^{\circ} \mathrm{C}$ with the monoclonal antibody II-II-6B3 against collagen type II diluted 1:20 in PBS, which was obtained from the Developmental Studies Hybridoma Bank (University of lowa, USA). After rinsing three times with PBS, slides were incubated for $45 \mathrm{~min}$ at $37^{\circ} \mathrm{C}$ with FITC-labeled anti-mouse IgG (Dianova, Hamburg, Germany) and diluted 1:800 in PBS. Slides were washed three times in PBS and briefly in distilled water. Specimens were embedded in Vectashield mounting medium (Vector, Burlingame, CA, USA) and analyzed using the fluorescence microscope Axioskop (Zeiss, Oberkochen, Germany). Control slides incubated with the secondary antibody alone were included.

Quantitative measurement gene expression by RT-qPCR analysis. Samples of $10 \mathrm{EBs}$ of different developmental stages up to 49 days after plating ( $5+49$ days) were collected, washed two times with PBS, and total RNA was isolated and reverse transcribed as described previosuly. ${ }^{5}$ For quantitative RT-PCR, we used TaqMan probes specific for the gene of interest (TIB Molbiol, Berlin, Germany), which were labeled with 6-FAM and had tetramethylrhodamine as a quencher. Aliquots of $1 \mu \mathrm{l}$ from the RT reactions were mixed with $19 \mu \mathrm{l}$ master mix consisting of $0.4 \mu \mathrm{l}$ TaqMan probe, $0.4 \mu \mathrm{l}$ gene-specific primer (see Supplementary Table 1), $10 \mu \mathrm{l}$ iQ Supermix (Bio-Rad, Munich, Germany) and $7.8 \mu \mathrm{l}$ sterile, steam sterilized and DEPC-treated water (Roth, Karlsruhe, Germany) per probe. The thermal cycling was performed using an iCycler iQ thermal cycler running with software version 3.1. (Bio-Rad) according to the manufacturer's instructions and the following thermal conditions: $95^{\circ} \mathrm{C}$ for 2 min followed by 50 cycles of $95^{\circ} \mathrm{C}$ for $40 \mathrm{~s}$, annealing temperature for $40 \mathrm{~s}$ (see Supplementary Table 1) and $72^{\circ} \mathrm{C}$ for $40 \mathrm{~s}$. Finally samples were heated to $95^{\circ} \mathrm{C}$ for $1 \mathrm{~min}$. For generation of standard curves, the PCR product was cloned into the vector PCR-TOPO (Invitrogen). Plasmid DNA was isolated using Qiagen-tip 100 anion-exchange columns (Qiagen, Hilden, Germany) and serially diluted in double-distilled water. Threshold cycles were adjusted to attain the highest possible correlation coefficient value for the standard curve provided by the manufacturer's software. According to their respective cycle numbers, the concentrations of unknown samples were deduced from the standard curve. Gene expression was determined relatively to expression of the housekeeping gene Gapdh. Initial experiments validating Sox9 as a Notch1 target gene (Figure 2), as well as Hes1, Hes5, Hey1 and Id3 measurements were carried out on a 7900HT Fast Real-Time PCR System (Applied Biosystems, Foster City, CA, USA) in 384-well PCR-plates (ABgene, Epsom, UK) using the TaqMan Gene Expression Assays-on-Demand assays Mm00448840_m1 (Sox9), Mm00492575_m1 (Id3) and Mm99999915_g1 (Gapdh) (Applied Biosystems) as described previously. ${ }^{13}$ Snai1 was measured using the Power SYBR green Master Mix (Applied Biosystems) with a primer concentration of $300 \mathrm{nM}$ (sequences see Supplementary Table 1) on a 7900HT Fast Real-Time PCR System (Applied Bioystems).

Luciferase assays and reporter plasmids. A mouse Sox9 luciferase pGL3 reporter construct comprising $4 \mathrm{~kb}$ before and $+315 \mathrm{bp}$ after transcription start site were used for luciferase experiments (kindly provided by Dr. Peter Koopman, Brisbane, Australia). ${ }^{47}$ The potential RBP-J-binding sites located at approximately $+40 \mathrm{bp}$ and $-325 \mathrm{bp}$ were mutated from TGTGGGAG to TGACATCG and from TTCACACG to TGTGTCAG, respectively, using the Quick Change technique using the Phusion Hot Start II Taq polymerase (Finnzymes/ Thermo Scientific). The primers used were $5^{\prime}$-GAAACTTCTGACATCGCGACAAC TTTACCAGTTT-3' (Sox9 BS1mut forward), 5'-AGTTGTCGCGATGTCAGAAGTTT CCAGGCAGTTC-3' (Sox9 BS1mut reverse), 5'-CACATCGGTGTGTCAGGAGAC CGTTCCAAAACTG-3' (Sox9 BS2mut forward) and 5'-ACGGTCTCCTGACACACC GATGTGTGTGTGTGTG-3' (Sox9 BS2mut reverse).

For transfection of luciferase reporter assays, $4 \times 10^{5} \mathrm{ESCs}$ were seeded in the presence or absence of $500 \mathrm{nM} \mathrm{OHT} \mathrm{in} \mathrm{24-well} \mathrm{plates} \mathrm{and} \mathrm{transfected} 24 \mathrm{~h}$ later using Fugene HD according to the manufacturer's instructions (Roche Diagnostics, Mannheim, Germany) with $0.9 \mu \mathrm{g}$ of the wild-type (Sox9-wt), BS1-mutated (Sox9BS1mut) or BS2-mutated construct (Sox9-BS2mut) and $0.1 \mu \mathrm{g}$ phRL-CMV plasmids (constitutive expression of Renilla luciferase for transfection efficiency control). Sixteen hours after transfection, measurements of luciferase activities were performed using the Dual Luciferase Kit (Promega, Madison, WI, USA) according to the manufacturer's instructions in a GloMax-96 Microplate Luminometer (Promega).

Data analysis and statistics. Data analysis was performed using the Sigma Plot 2000 software (Jandel, Corte Madeira, CA, USA). The Prism 5 software (GraphPad Software, La Jolla, CA, USA) and the R software (http://www. r-project.org) or SPSS 15 (http://www.SPSS.com; SPSS Inc., Chicago, IL, USA) were used for statistical analysis. For statistical analysis, one- or two-way analysis of variance was performed followed by Tukey's range test and Bonferroni's post-tests, respectively. Error bars present S.E.M. For statistical analysis of qPCR results, raw $\Delta \mathrm{Ct}$ values were used.

Acquisition and processing of images. Slides were analyzed using the fluorescence microscope Axioskop (Zeiss) equipped with a three CCD color video camera (Sony, Cologne, Germany) using the acquisition software Axiovision (Zeiss). Figures were assembled using the Corel Draw software (Corel, Ottawa, Canada).

\section{Conflict of Interest}

The authors declare no conflict of interest.

Acknowledgements. We are very grateful to A Tiedtke and B Andresen for their skillful technical assistance and to Peter Koopman for providing the mouse Sox9 luciferase pGL3 reporter construct. The II-II 6B3 monoclonal antibody developed by TF Linsenmayer was obtained from the Developmental Studies Hybridoma Bank developed under the auspices of the NICHD and maintained by the University of lowa, Department of Biological Sciences, lowa City, IA52242. This work was supported by funding from the DFG SFB 415 project B8 to UJ and from Intermed Service GmbH, Geesthacht to JK and JR. 


\section{Author contributions}

Ralf Haller: Collection and/or assembly of data, data analysis and interpretation, drafted manuscript and final approval of manuscript. Ralf Schwanbeck: Collection and/or assembly of data, data analysis and interpretation, provision of study materials, drafted and revised manuscript and final approval of manuscript. Simone Martini: Collection and/or assembly of data, provision of study materials, revised manuscript and final approval of manuscript. Kristina Bernoth: Collection and/or assembly of data, provision of study materials, revised manuscript and final approval of manuscript. Jan Kramer: Data analysis and interpretation, drafted manuscript, financial support and final approval of manuscript. Ursula Just: Concept and design, data analysis and interpretation, provision of study materials, drafted and revised manuscript, financial support and final approval of manuscript. Jürgen Rohwedel: Concept and design, data analysis and interpretation, financial support, drafted and revised manuscript and final approval of manuscript.

1. Artavanis-Tsakonas $S$, Rand MD, Lake RJ. Notch signaling: cell fate control and signal integration in development. Science 1999; 284: 770-776.

2. Kopan $\mathrm{R}$, llagan $M X$. The canonical Notch signaling pathway: unfolding the activation mechanism. Cell 2009; 137: 216-233.

3. Zanotti S, Canalis E. Notch and the skeleton. Mol Cell Biol 2010; 30: 886-896.

4. Watanabe N, Tezuka Y, Matsuno K, Miyatani S, Morimura N, Yasuda M et al. Suppression of differentiation and proliferation of early chondrogenic cells by Notch. J Bone Miner Metab 2003; 21: 344-352.

5. Hegert C, Kramer J, Hargus G, Muller J, Guan K, Wobus AM et al. Differentiation plasticity of chondrocytes derived from mouse embryonic stem cells. J Cell Sci 2002; 115 4617-4628.

6. Kramer J, Hegert C, Guan K, Wobus AM, Muller PK, Rohwedel J. Embryonic stem cellderived chondrogenic differentiation in vitro: activation by BMP-2 and BMP-4. Mech Dev 2000; 92: 193-205.

7. Hayes AJ, Dowthwaite GP, Webster SV, Archer CW. The distribution of Notch receptors and their ligands during articular cartilage development. J Anat 2003; 202: 495-502.

8. Mead TJ, Yutzey KE. Notch pathway regulation of chondrocyte differentiation and proliferation during appendicular and axial skeleton development. Proc Natl Acad Sci USA 2009; 106: 14420-14425.

9. Dong Y, Jesse AM, Kohn A, Gunnell LM, Honjo T, Zuscik MJ et al. RBPjkappa-dependent Notch signaling regulates mesenchymal progenitor cell proliferation and differentiation during skeletal development. Development 2010; 137: 1461-1471.

10. Ijuin K, Nakanishi K, Ito K. Different downstream pathways for Notch signaling are required for gliogenic and chondrogenic specification of mouse mesencephalic neural crest cells Mech Dev 2008; 125: 462-474.

11. Oldershaw RA, Tew SR, Russell AM, Meade K, Hawkins R, McKay TR et al. Notch signaling through Jagged- 1 is necessary to initiate chondrogenesis in human bone marrow stromal cells but must be switched off to complete chondrogenesis. Stem Cells 2008; 26 666-674.

12. Iso T, Kedes L, Hamamori Y. HES and HERP families: multiple effectors of the Notch signaling pathway. J Cell Physiol 2003; 194: 237-255

13. Meier-Stiegen F, Schwanbeck R, Bernoth K, Martini S, Hieronymus T, Ruau D et al. Activated Notch1 target genes during embryonic cell differentiation depend on the cellular context and include lineage determinants and inhibitors. PLoS One 2010; 5: e11481.

14. Wright E, Hargrave MR, Christiansen J, Cooper L, Kun J, Evans T et al. The Sry-related gene Sox9 is expressed during chondrogenesis in mouse embryos. Nat Genet 1995; 9 $15-20$.

15. Lefebvre V, Huang W, Harley VR, Goodfellow PN, de Crombrugghe B. SOX9 is a potent activator of the chondrocyte-specific enhancer of the pro alpha1(II) collagen gene. Mol Cell Biol 1997; 17: 2336-2346.

16. Liu Y, Li H, Tanaka K, Tsumaki N, Yamada Y. Identification of an enhancer sequence within the first intron required for cartilage-specific transcription of the alpha2(XI) collagen gene. J Biol Chem 2000; 275: 12712-12718.

17. Sekiya I, Tsuji K, Koopman P, Watanabe H, Yamada Y, Shinomiya K et al. SOX9 enhances aggrecan gene promoter/enhancer activity and is up-regulated by retinoic acid in cartilage-derived cell line, TC6. J Biol Chem 2000; 275: 10738-10744.

18. Wagner T, Wirth J, Meyer J, Zabel B, Held M, Zimmer J et al. Autosomal sex reversal and campomelic dysplasia are caused by mutations in and around the SRY-related gene SOX9. Cell 1994; 79: 1111-1120.

19. Bi W, Huang W, Whitworth DJ, Deng JM, Zhang Z, Behringer RR et al. Haploinsufficiency of Sox9 results in defective cartilage primordia and premature skeletal mineralization. Proc Natl Acad Sci USA 2001; 98: 6698-6703.

20. Bi W, Deng JM, Zhang Z, Behringer RR, de Crombrugghe B. Sox 9 is required for cartilage formation. Nat Genet 1999; 22: 85-89.
21. Akiyama $\mathrm{H}$, Chaboissier MC, Martin JF, Schedl A, de Crombrugghe B. The transcription factor Sox9 has essential roles in successive steps of the chondrocyte differentiation pathway and is required for expression of Sox5 and Sox6. Genes Dev 2002; 16 : 2813-2828

22. Akiyama H, Kim JE, Nakashima K, Balmes G, Iwai N, Deng JM et al. Osteochondroprogenitor cells are derived from Sox9 expressing precursors. Proc Natl Acad Sci USA 2005; 102: 14665-14670.

23. Mori-Akiyama $\mathrm{Y}$, Akiyama $\mathrm{H}$, Rowitch $\mathrm{DH}$, de Crombrugghe B. Sox9 is required for determination of the chondrogenic cell lineage in the cranial neural crest. Proc Natl Acad Sci USA 2003; 100: 9360-9365.

24. Akiyama H, Stadler HS, Martin JF, Ishii TM, Beachy PA, Nakamura T et al. Misexpression of Sox9 in mouse limb bud mesenchyme induces polydactyly and rescues hypodactyly mice. Matrix Biol 2007; 26: 224-233.

25. Hargus G, Kist R, Kramer J, Gerstel D, Neitz A, Scherer G et al. Loss of Sox9 function results in defective chondrocyte differentiation of mouse embryonic stem cells in vitro. Int $J$ Dev Biol 2008; 52: 323-332

26. Schroeder T, Meier-Stiegen F, Schwanbeck R, Eilken $\mathrm{H}$, Nishikawa S, Hasler $\mathrm{R}$ et al. Activated Notch1 alters differentiation of embryonic stem cells into mesodermal cell lineages at multiple stages of development. Mech Dev 2006; 123: 570-579.

27. Schroeder T, Just U. Notch signalling via RBP-J promotes myeloid differentiation. EMBO J 2000; 19: 2558-2568

28. Schwanbeck R, Martini S, Bernoth K, Just U. The Notch signaling pathway: molecular basis of cell context dependency. Eur J Cell Biol 2011; 90: 572-581.

29. Nemir M, Croquelois A, Pedrazzini T, Radtke F. Induction of cardiogenesis in embryonic stem cells via downregulation of Notch1 signaling. Circ Res 2006; 98: 1471-1478.

30. Zhou Y, Snead ML. Derivation of cranial neural crest-like cells from human embryonic stem cells. Biochem Biophys Res Commun 2008; 376: 542-547.

31. Cormier S, Vandormael-Pournin S, Babinet C, Cohen-Tannoudji M. Developmental expression of the Notch signaling pathway genes during mouse preimplantation development. Gene Expr Patterns 2004; 4: 713-717.

32. Karlsson C, Jonsson M, Asp J, Brantsing C, Kageyama R, Lindahl A. Notch and HES5 are regulated during human cartilage differentiation. Cell Tissue Res 2007; 327: 539-551.

33. Pedrazzini T. Control of cardiogenesis by the notch pathway. Trends Cardiovasc Med 2007; 17: 83-90.

34. Schroeder T, Fraser ST, Ogawa M, Nishikawa S, Oka C, Bornkamm GW et al. Recombination signal sequence-binding protein Jkappa alters mesodermal cell fate decisions by suppressing cardiomyogenesis. Proc Natl Acad Sci USA 2003; 100 : 4018-4023.

35. Lowell S, Benchoua A, Heavey B, Smith AG. Notch promotes neural lineage entry by pluripotent embryonic stem cells. PLOS Biol 2006; 4: e121.

36. Cornell RA, Eisen JS. Notch in the pathway: the roles of Notch signaling in neural crest development. Semin Cell Dev Biol 2005; 16: 663-672.

37. Cheung M, Briscoe J. Neural crest development is regulated by the transcription factor Sox9. Development 2003; 130: 5681-5693.

38. Alagille D, Odievre M, Gautier M, Dommergues JP. Hepatic ductular hypoplasia associated with characteristic facies, vertebral malformations, retarded physical, mental, and sexual development, and cardiac murmur. J Pediatr 1975; 86: 63-71.

39. Muto A, lida A, Satoh S, Watanabe S. The group E Sox genes Sox8 and Sox9 are regulated by Notch signaling and are required for Muller glial cell development in mouse retina. Exp Eye Res 2009; 89: 549-558.

40. Fischer A, Gessler M. Delta-Notch - and then? Protein interactions and proposed modes of repression by Hes and Hey bHLH factors. Nucleic Acids Res 2007; 35: 4583-4596.

41. Karlsson C, Brantsing C, Kageyama R, Lindahl A. HES1 and HES5 are dispensable for cartilage and endochondral bone formation. Cells Tissues Organs 2010; 192: 17-27.

42. Salie R, Kneissel M, Vukevic M, Zamurovic N, Kramer I, Evans G et al. Ubiquitous overexpression of Hey1 transcription factor leads to osteopenia and chondrocyte hypertrophy in bone. Bone 2010; 46: 680-694.

43. Grogan SP, Olee T, Hiraoka K, Lotz MK. Repression of chondrogenesis through binding of notch signaling proteins HES-1 and HEY-1 to N-box domains in the COL2A1 enhancer site. Arthritis Rheum 2008; 58: 2754-2763.

44. Steventon B, Carmona-Fontaine C, Mayor R. Genetic network during neural crest induction: from cell specification to cell survival. Semin Cell Dev Biol 2005; 16: 647-654.

45. de Frutos CA, Vega S, Manzanares M, Flores JM, Huertas H, Martinez-Frias ML et al. Snail1 is a transcriptional effector of FGFR3 signaling during chondrogenesis and achondroplasias. Dev Cell 2007; 13: 872-883.

46. Mukhopadhyay P, Rezzoug F, Webb CL, Pisano MM, Greene RM. Suppression of chondrogenesis by Id helix-loop-helix proteins in murine embryonic orofacial tissue. Differentiation 2009; 77: 462-472.

47. Kanai $Y$, Koopman $P$. Structural and functional characterization of the mouse Sox 9 promoter: implications for campomelic dysplasia. Hum Mol Genet 1999; 8: 691-696. 